\title{
SECAGEM DE GRANULADO MINERAL: EXPLORAÇÃO DA EQUAÇÃO DE ARRHENIUS COMO FERRAMENTA PARA ESTIMAR A ENERGIA DE ATIVAÇÃO E A DIFUSIVIDADE EFETIVA
}

\author{
T. C. A. SOUZA e H. PERAZZINI \\ Universidade Federal de Itajubá, Instituto de Recursos Naturais \\ E-mail para contato: perazzini@unifei.edu.br
}

\begin{abstract}
RESUMO - No presente trabalho, é apresentado um estudo da cinética de secagem de um granulado mineral poroso. $\mathrm{O}$ material foi caracterizado por meio da determinação de suas principais propriedades físicas e estruturais. O modelo difusivo reparametrizado foi utilizado para ajustar dados experimentais de umidade em função do tempo. Como as primeiras propostas de ajuste não foram satisfatórias, uma nova estratégia foi adotada, realizando-se novos estudos de simulação para duas diferentes zonas de secagem, denominadas: zona seca e zona úmida. Com base nos resultados obtidos, verificou-se que a energia de ativação é maior para a zona seca e menor pra a zona úmida, enquanto que o inverso foi observado para a difusividade efetiva.
\end{abstract}

\section{INTRODUÇÃO}

A secagem é uma das operações unitárias mais utilizadas atualmente, fazendo parte dos mais diversos processos industriais. Entretanto, para que a secagem ocorra, são necessárias para alguns materiais altas temperaturas e elevada velocidade de ar, o que acarreta custos operacionais e energéticos muito altos, aumentando consideravelmente o valor do produto final, podendo até inviabilizar sua comercialização. Assim, para que a secagem seja economicamente viável é preciso otimizar o processo, estudando os mecanismos de transporte envolvidos e as características do material a ser seco. Apesar de ser um processo já consolidado e muito utilizado em indústrias, a secagem raramente é operada em sua máxima eficiência. Estudos de cinética de secagem, por sua vez, auxiliariam na otimização do processo, minimizando o consumo de insumos e os gastos energéticos envolvidos. Esta pesquisa teve como objetivo analisar a cinética de secagem de um granulado mineral, que por apresentar excelente porosidade e elevada capacidade de adsorção, possui potencial para ser considerado uma ótima alternativa para a composição do recheio de colunas de adsorção, por exemplo.

\section{MATERIAIS E MÉTODOS}

\subsection{Material Particulado}

O material estudado é um granulado mineral branco composto de gesso, ligantes e fibras minerais, inerte e atóxico que possui o núcleo poroso, fazendo com que a sua 
capacidade de absorção e adsorção seja alta. O granulado foi estudado em três granulometrias: partículas finas, médias e grossas, com diâmetros de $0,5 \mathrm{~mm}, 1 \mathrm{~mm}$ e $2 \mathrm{~mm}$, respectivamente. As propriedades obtidas durante a caracterização dos sólidos estão disponíveis na Tabela 1.

Tabela 1 - Propriedades estimadas para o granulado mineral.

\begin{tabular}{|c|c|c|c|c|c|c|}
\hline Método & Picnometria & Gravimetria & Cálculos & $\begin{array}{c}\text { Análise de } \\
\text { Imagens }\end{array}$ & $\begin{array}{c}\text { Análise de } \\
\text { Imagens }\end{array}$ & BET \\
\hline Propriedade & $\begin{array}{c}\text { Massa } \\
\text { Específica } \\
{\left[\mathrm{g} / \mathrm{cm}^{3}\right]}\end{array}$ & $\begin{array}{c}\text { Massa } \\
\text { Específica do } \\
\text { Meio }\left[\mathrm{Kg} / \mathrm{m}^{3}\right]\end{array}$ & $\begin{array}{c}\text { Porosidade } \\
\text { do Meio }[-]\end{array}$ & Área $\left[\mathrm{mm}^{2}\right]$ & $\begin{array}{c}\text { Esfericidade } \\
{[-]}\end{array}$ & $\begin{array}{c}\text { Tamanho } \\
\text { Médio dos } \\
\text { Poros [nm] }\end{array}$ \\
\hline Valor médio & 1,603 & 809,183 & 0,495 & 6,733 & 0,869 & 0,849 \\
\hline
\end{tabular}

\subsection{Unidade Experimental de Secagem}

Os experimentos de secagem do granulado mineral foram realizados em uma unidade experimental de secagem, que consiste de uma ventoinha acoplada a um tubo horizontal de PVC. Ar ambiente foi aquecido por meio do contato com resistências elétricas e sua temperatura aferida por um termômetro de mercúrio, conforme representado na Figura 1.

Figura 1- Unidade experimental de secagem.

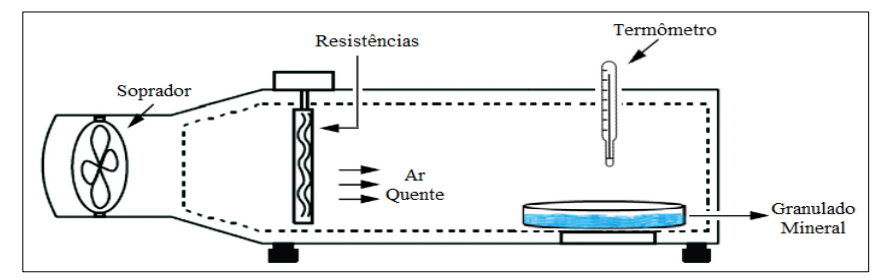

\subsection{Metodologia}

Após submeter o material a um processo de umidificação, iniciou-se o procedimento de secagem, sendo que em cada experimento utilizou-se condições de operacionais diferentes. Um total de doze experimentos foram realizados, modificando-se apenas variáveis como temperatura de secagem e espessura da camada de material, nas seguintes faixas, para cada diâmetro de partícula: $45<\mathrm{T}<60\left[{ }^{\circ} \mathrm{C}\right]$ e $1<\mathrm{L}<0,5[\mathrm{~cm}]$. Nos experimentos, a pesagem dos sólidos foi feita constantemente em uma balança analítica, até que a massa do material deixasse de variar. Com o fim do experimento o granulado foi levado a uma estufa a $105^{\circ} \mathrm{C}$ para determinação de seu peso seco.

\section{TRATAMENTO DOS DADOS}

Os dados de secagem foram ajustados com base no modelo difusivo considerando duas abordagens: $\mathrm{Bi}_{\mathrm{m}} \rightarrow \infty$ e $\mathrm{Bi}_{\mathrm{m}} \rightarrow 0$, resultando nas soluções analíticas representadas pelas Equações (1) e (2), respectivamente com base em uma série de hipóteses (CRANK, 1975).

$$
X^{*}=\frac{\bar{X}-X_{e q}}{X_{0}-X_{e q}}=\frac{8}{\pi^{2}} \sum_{n=0}^{\infty} \frac{1}{(2 n+1)^{2}} \exp \left[-(2 n+1)^{2} \pi^{2} \frac{D_{e f f}}{4 L^{2}} t\right]
$$




$$
X^{*}=\frac{\bar{X}-X_{e q}}{X_{0}-X_{e q}}=2 \sum_{n=1}^{\infty} \frac{B i_{m}{ }^{2}}{\lambda_{n}^{2}\left(\lambda_{n}^{2}+B i_{m}{ }^{2}+B i_{m}\right)} \exp \left(-\lambda_{n}^{2} \frac{D_{e f f}}{4 L^{2}} t\right)
$$

Para a Equação (2), as raízes $\lambda_{\mathrm{n}}$ são determinadas pela seguinte equação transcendental:

$$
B i_{m}=\lambda_{n} \operatorname{tg}\left(\lambda_{n}\right)
$$

A difusividade efetiva $\left(D_{\text {eff }}\right)$ é usualmente tratada pela literatura como função da temperatura, segundo uma correlação do tipo Arrhenius:

$$
D_{e f f}=D_{0} \exp \left(-\frac{E_{a}}{R T}\right)
$$

Entretanto, foi demonstrado por Kiranoudis et al. (1995) que o fator de Arrhenius $\mathrm{D}_{0}$ e a energia de ativação para a difusão de umidade $\left(E_{a}\right)$ são propriedades fortemente correlacionadas. Entende-se, assim, que o valor estimado da difusividade efetiva é devido a uma combinação destes dois parâmetros. Em estudos envolvendo cinética química, Mezaki e Kittrell (1967) verificaram a necessidade de reparametrização da correlação de Arrhenius, uma vez verificada a não-linearidade dos parâmetros $D_{0}$ e $E_{a}$ e resultados inadequados na estimação. Freire et al. (1995) efetuaram a reparametrização, resultando na seguinte transformação da Equação (4):

$$
D_{\text {eff }}=\exp (\beta) \exp \left[-\left(\frac{1}{T}-\frac{1}{T^{*}}\right) \exp (\gamma)\right]
$$

A substituição da Equação (5) nas soluções analíticas do modelo difusivo e fazendo T* $=273,15 \mathrm{~K}$, resulta em um modelo com novos graus de liberdade e um novo conjunto de parâmetros a serem estimados.

\section{RESULTADOS}

\subsection{Cinética de Secagem}

As curvas apresentadas na Figura 2 demonstram que para o sistema particulado: o tempo de secagem diminui com o aumento da temperatura ou com a redução da espessura da camada de sólidos (L).

Figura 2 - Comparação entre as curvas de cinética de secagem obtidas.

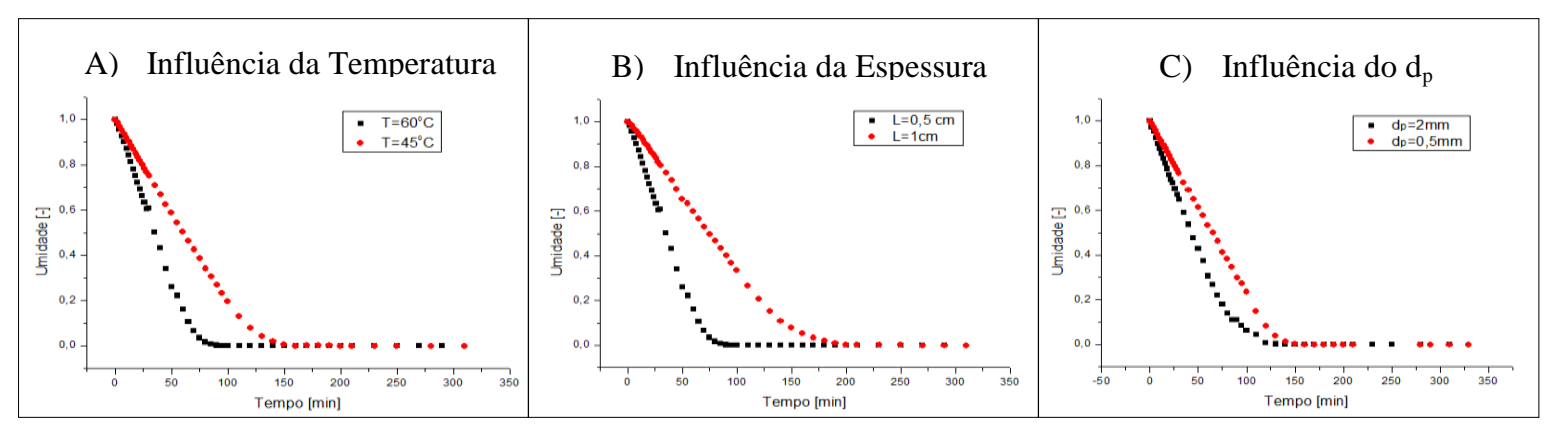


Além disso, verificou-se também que as partículas finas demoram mais para secar do que as grossas, fato que pode ser justificado pelo maior "empacotamento" que existe na camada de partículas finas, fazendo com que o espaço entre estas partículas seja muito pequeno, o que dificulta a migração da água de dentro da camada para sua superfície, e assim ser transferida para a corrente de ar na forma de vapor.

\subsection{Ajuste dos Modelos}

Na Figura 3 observa-se a comparação típica entre dados observados e preditos pelas Equações (1) e (2) e verifica-se que, apesar da semelhança entre as duas curvas que o modelo previu, a aproximação foi melhor para a simulação em que $\mathrm{Bi}_{\mathrm{m}} \rightarrow 0$, indicando que é a convecção o mecanismo dominante no processo. Isto pode ser melhor constatado a partir dos valores de $R^{2}$, que foi 0,923 para a Equação (1) e 0,976 para a Equação (2). Resultados similares foram verificados para as demais condições operacionais estudadas.

Figura 3 - Comparação entre dados preditos e observados para diferentes condições de contorno do modelo difusivo.

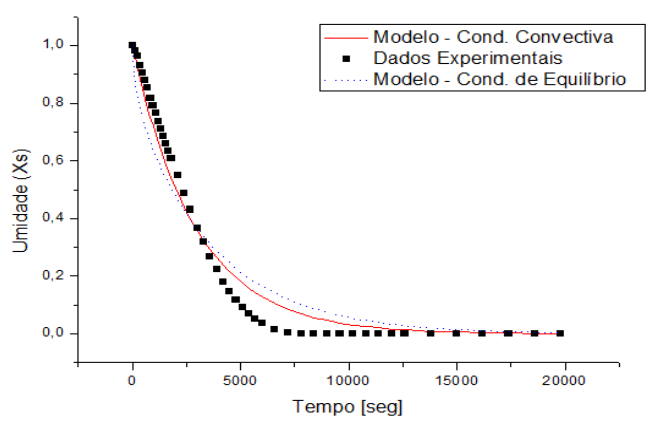

Devido ao fato das duas curvas obtidas por meio da simulação com o modelo difusivo terem ficado muito próximas, foi necessário buscar outras técnicas para melhorar a eficiência do ajuste. Com base em Fiorentini et al. (2015), a nova estratégia adotada para melhorar o ajuste dos modelos consistiu em dividir os dados de umidade em função do tempo em duas zonas de secagem, zona seca e zona úmida, e ajustar o modelo difusivo reparametrizado para cada zona. Para a definição de cada zona, determinou-se o tempo crítico considerando duas abordagens diferentes. Em um primeiro momento, utilizou-se o modelo descrito por Sander $e t$ al. (2010), baseado na Equação 6, para a determinação do tempo $t_{k}$, que indica o tempo em que ocorre a transição entre os períodos de taxa constante e decrescente, correspondendo ao tempo em que o sólido atinge a umidade crítica.

$$
\frac{X(t)-X_{e q}}{X_{0}-X_{e q}}=\exp \left[-\left(t / t_{k}\right)^{n}\right]
$$

O método de linearização dos dados (KEEY, 1978) também foi utilizado para encontrar valores de tempo crítico. Adotou-se a média entre todos os resultados de $t_{c}$ e $t_{k}$ para efetuar a divisão dos dados de secagem. Os dados de umidade que antecederam o tempo crítico médio $\left(t_{c}\right)$ constituíram a parte da curva chamada de zona úmida, pois nesta etapa do processo o material ainda apresentava umidade em quantidade significativa. Já os valores de umidade que estavam após $t_{c}$ formaram a segunda parte da curva, chamada de zona seca. $\mathrm{O}$ 
ajuste de modelos foi feito novamente em MatLab, mas agora com os dados divididos em duas partes e implementados separadamente. Como se pode verificar na Figura 4, a aproximação do modelo foi muito satisfatória, resultando em um $\mathrm{R}^{2}$ médio igual a 0,994 .

Figura 4 - Simulação para a zona úmida e zona seca para $\mathrm{T}=45^{\circ} \mathrm{C}, \mathrm{L}=0,5 \mathrm{~cm}$ e $d_{p}=2 \mathrm{~mm}$.

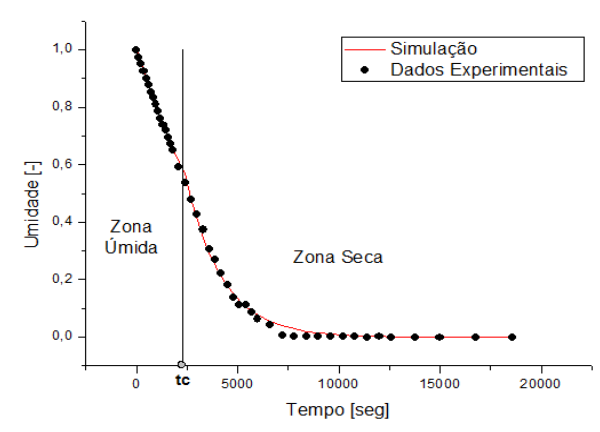

Como se esperava os parâmetros estimados pelo modelo foram diferentes para a zona úmida e para a zona seca, conforme apresentado na Tabela 2. Na zona úmida ainda existe água disponível, por isso a energia necessária para que a água consiga evaporar é menor. Já na zona seca, há menores conteúdos de umidade, o que torna mais difícil a migração de umidade neste estágio do processo, acarretando em uma $\mathrm{E}_{\mathrm{a}}$ maior do que na zona úmida.

Tabela 2 - Parâmetros estimados para as condições $\mathrm{T}=45^{\circ} \mathrm{C}, \mathrm{L}=0,5 \mathrm{~cm}$ e $d_{p}=2 \mathrm{~mm}$.

\begin{tabular}{|c|c|c|c|c|c|c|c|}
\hline Zona & $\mathbf{D}_{\mathbf{0}}\left[\mathbf{m}^{\mathbf{2}} / \mathbf{s}\right]$ & $\boldsymbol{\Upsilon}$ & $\boldsymbol{\beta}$ & $\mathbf{D}_{\text {eff }}\left[\mathbf{m}^{\mathbf{2}} / \mathbf{s}\right]$ & $\mathbf{E a}[\mathbf{k J} / \mathbf{m o l}]$ & $\mathbf{B i}_{\mathbf{m}}$ & $\mathbf{R}^{\mathbf{2}}$ \\
\hline Úmida & $6,38 \mathrm{E}-06$ & 7,30 & $-17,362$ & $6,19 \mathrm{E}-08$ & 12,264 & 0,101 & 0,997 \\
\hline Seca & $3,40 \mathrm{E}-04$ & 7,72 & $-16,243$ & $2,84 \mathrm{E}-07$ & 18,750 & 0,088 & 0,991 \\
\hline
\end{tabular}

Por outro lado, as estimativas da difusividade efetiva mostram que este parâmetro foi menor durante a zona úmida, fato que pode ser justificado quando se relaciona a $\mathrm{D}_{\text {eff }}$ com a temperatura, ou seja, o produto é aquecido gradativamente por meio do ar de secagem, por isso, na zona úmida, os sólidos secam na temperatura de bulbo úmido do ar, como conseqüência da presença do calor latente, resultando em uma $\mathrm{D}_{\text {eff }}$ menor. Já na zona seca, onde prevalece o calor sensível e o corpo utiliza toda energia disponível para elevar a sua temperatura, o produto ficou em contato com o ar quente por mais tempo e por isso apresentou temperatura próxima da do ar, acarretando no aumento da $\mathrm{D}_{\text {eff. }}$.

\section{CONCLUSÕES}

A partir da modelagem da secagem empregando-se soluções analíticas do modelo difusivo foi possível concluir que a convecção é o mecanismo dominante. A estratégia de dividir o processo em duas etapas e simulá-las separadamente nos permitiu verificar que parâmetros como difusividade efetiva e energia de ativação não permaneceram constantes durante todo o processo. A energia de ativação foi maior para a zona seca, isto é, para o período da secagem no qual o sólido possuiu menor umidade e, para a zona úmida, a energia de ativação foi menor, indicando uma maior mobilidade das moléculas de água neste estágio do processo. A difusividade efetiva, do contrário, foi maior para a zona seca, e menor para a zona úmida. Os resultados sugeriram que estes parâmetros não devem ser tratados como constantes durante a modelagem do processo. 


\section{NOMENCLATURA}

$\mathrm{Bi}_{\mathrm{m}}$ : número de Biot para transferência de massa [-]

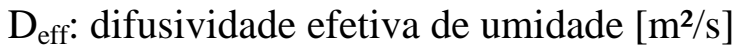

$d_{p}$ : diâmetro da partícula [mm]

$\mathrm{D}_{0}$ : fator pré-exponencial de Arrhenius $\left[\mathrm{m}^{2} / \mathrm{s}\right]$

$\mathrm{E}_{\mathrm{a}}$ : energia de ativação $[\mathrm{kJ} / \mathrm{mol}]$

L: espessura do sólido [m]

$\mathrm{n}$ : número de termos da série infinita [-]

$\mathrm{R}$ : constante dos gases ideais $[\mathrm{kJ} / \mathrm{mol} \cdot \mathrm{K}]$

$\mathrm{T}^{*}$ : temperatura de referência $[\mathrm{K}]$

$\mathrm{T}$ : temperatura do ar $[\mathrm{K}]$

$\mathrm{t}$ : tempo de secagem [s]

$\mathrm{X}^{*}$ : umidade adimensional [-]

$\mathrm{X}_{\mathrm{eq}}$ : umidade de equilíbrio $[\mathrm{kg} / \mathrm{kg}]$

$X$ : umidade em base seca $[\mathrm{kg} / \mathrm{kg}]$

$\mathrm{X}_{0}$ : umidade inicial $[\mathrm{kg} / \mathrm{kg}]$

$\overline{\mathrm{X}}$ : umidade média $[\mathrm{kg} / \mathrm{kg}]$

$\beta$ : parâmetro da equação reparametrizada que representa a variação de $\mathrm{D}_{\text {eff }} \operatorname{com} \mathrm{T}[-]$

$\gamma$ : parâmetro da equação reparametrizada que representa a variação de $\mathrm{D}_{\text {eff }} \operatorname{com} \mathrm{T}[-]$

$\lambda$ : raízes da equação transcendental [-]

\section{REFERÊNCIAS}

BARROZO, M.A.S., SARTORI, D.J.M., FREIRE, J.T., 2004. A study of the statistical discrimination of the drying kinetics equations. Food Bioprod. Process. 82, 219-225.

CRANK, J. The mathematics of diffusion. 2. ed. Oxford: Claredon Press, p. 414, 1975.

FIORENTINI, C.; DEMARCHI, S. M.; RUIZ, N. A. Q.; IRIGOYEN, R. M. T.; GINER, S. A.; Arrhenius Activation Energy for Water Diffusion During Drying of Tomato Leathers: The Concept of Characteristic Product Temperature. Biosyst. Eng., v.132, p. 39-46, 2015.

KEEY, R. B. Introduction to industrial drying operations. Oxford: Pergamon Press, p. 376, 1978.

KIRANOUDIS, C. T.; MAROULIS, Z.B.; MARINOS-KOURIS, D. 1995. Heat and mass transfer model building in drying with multi-response data. Int. J. Heat Mass Transfer, 38(3), 463-480.

MEZAKI, R.; KITTRELL, J.R. Parametric sensitivity in fitting nonlinear kinetic models. Ind. Eng. Chem. Res. 1967, 59 (5), 63-69.

SANDER, A.; KARDUM, J.P.; GLASNOVIC, A. Drying of Solids; Estimation of The Mathematical Model Parameters.The Can. J. of Chem. Eng., v.88, p. 822-829, 2010. 